\title{
SVM Relevance Feedback in HSV Quantization for CBIR
}

\author{
Jasman Pardede ${ }^{1,2 *}$, Benhard Sitohang², Saiful Akbar², Masayu Leylia Khodra² \\ ${ }_{1}^{1}$ Department of Informatics Engineering, Institut Teknologi Nasional (Itenas) Bandung, Bandung, Indonesia. \\ ${ }^{2}$ School of Electrical and Informatics, Institut Teknologi Bandung (ITB), Bandung, Indonesia. \\ * Corresponding author. Tel.: +62(22)7272215; email: jasman@itenas.ac.id \\ Manuscript submitted Juny 10, 2018; accepted August 29, 2018. \\ doi: $10.17706 /$ jcp.13.12.1366-1384
}

\begin{abstract}
In this research have implemented SVM relevance feedback technique in HSV quantization for CBIR. The proposed technique has two stages. The first stage performs image retrieval process based on results of distance measurement. The distance measurement used is Jeffrey Divergence with threshold 0.15. The second stage is image retrieval process based on SVM RF prediction model. The SVM RF model is formed based on user-provided feedback images. The users' feedback images are labeled as positive and others are negative. The purpose of this study is to determine the best value of the constant $C$ on the linear kernel and the best value of the constant $(\mathrm{C}, \mathrm{G})$ on the RBF kernel The best value of the constants provided generates the best model of SVM RF in the HSV Quantization method so that improve the performance of the CBIR system. Performance measurements are evaluated based on precision, recall, F-measure, and accuracy values. Based on the results of experiments that conduct on Wang dataset obtained that $(C, G)=\left(2^{2.725}, 2^{2.725}\right)$ is the best value on the RBF kernel. While $C=2^{5.275}$ is the best value on SVM RF using linear kernel. The best of SVM RF technique is SVM RF using RBF kernel of second feedback. The SVM RF using RBF kernel increases the average precision value by $3.02 \%$, the average recall value increasing amount $171.48 \%$, the average F-Measure value increasing amount $80.34 \%$, while the average accuracy value increasing amount $1.99 \%$ compared with the baseline. The SVM RF using RBF kernel obtains the best value on both the average recall value and the average F-Measure value compared to the state-of-the-art.
\end{abstract}

Key words: SVM, relevance feedback, HSV Quantization, CBIR.

\section{Introduction}

A system that facilitates users to retrieve images based on similarity content called Content-Based Image Retrieval (CBIR) [1]-[4]. The image content is represented by the features or attributes of the digital image [5]. Preliminary, the CBIR system was implemented using features of color, shape, texture or their combination [6]-[8]. Until now, various methods have been implemented on the CBIR system i.e.: GCE-SVM [9], ENN-SA [10], ENN-BR [11], CO-FSS [12], ASI-GTR [13], CTF [14], and others. However, at this time, the problems that are often encountered with the CBIR system are a semantic gap and computational complexity [15]-[19]. The semantic gaps can be caused by several things, i.e. a failure of the low-level feature takes the location of the image details, unavailability of image semantic representation, an ambiguity toward the given image query, and the user is not involved in the assessment of an image [15]-[17].

Semantic gaps can be reduced by involving humans as part of the feedback process, called Relevance Feedback (RF) [1], [20]-[25]. In the CBIR system with RF, it will encourage the user to provide an 
assessment of the images that retrieved. Algorithms implemented in the CBIR system with RF must also be able to learn and understand users' information needs based on user feedback [23], [26]. The feedback image is rated as positive or negative, highly relevant, relevant or irrelevant, partially relevant, and highly irrelevant [20], [22]. The users' feedback image is used for query improvements or the establishment of a classification model. Feedback process will be done repeatedly until the user satisfied.

In general, the RF techniques can be grouped into two categories, namely passive learning and active learning. Some of the passive learning RF techniques that well known are MARS [27]; MindReader [28], and several re-weighting approaches [29], [30], and others. In the active learning RF, the proposed algorithm or method should be able to identify the image examples so that can be very helpful for understanding the users' information needs. Unlike the passive RF, the system only enough presents images that have a high relevance value to the user. One of the active learning RF approaches in the CBIR system is Support Vector Machine active learning (SVM active learning) [31].

In conventional SVM active learning has problems in terms of the number of training data. The conventional SVM active learning is only designed to be able to select a single example to each of learning iteration [32]. In this research, implement SVM active learning with feature extract from HSV Quantization which is designed to be able to provide feedback image of the varying amount of image feedback. To accomplish the small and unbalanced amount of training data from the users' feedback is done by generating the new training data based on the generation of a new image from the selected image features randomly. The results of this study showed that SVM active learning can reduce the semantic gap.

This paper is organized into four sections. In section 2, describe about CBIR, HSV Quantization, Support Vector Machine, Support Vector Machine Relevance Feedback, and Wang Dataset. In section 3, presents experimental results and discussions conduct to Wang dataset. In section 4 is given the conclusion of the result of research.

\section{Literature Study}

\subsection{CBIR}

The Content-Based Image Retrieval (CBIR) is a tool that facilitates the user to retrieve images of the image collection or the image database according to the users' query image [1], [3], [33]-[35]. The CBIR retrieves images of the images collection based on their similarity. The similarity with the image can be obtained mathematically based on the features, attributes or parameters of the digital image [36], [37]. To obtain the query image similarity with the existing image of the image collection is determined by using the distance measurement [36] or using the particular classification techniques [38].

In general, the CBIR system has two feature extractions, i.e. the off-line features extraction and the online features extraction [29]. The off-line features extraction is used to extract the features of each image of the database automatically and then store it in the database feature. While the online feature extraction is used to extract the features of the query image when searching for specific images. To obtain images that have a better similarity is done by sorting the results of similarity measurements in accordance with the degree of similarity with the image. There are some similarity measurements that commonly used in the CBIR system, i.e. Euclidean Distance, Cramer-von Mises Divergence, Manhattan Distance, Cosine Similarity, Chi-Square Dissimilarity, Jeffrey Divergence, Pearson Correlation Coefficient, and Mahalanobis Distance [36], [37]. The results of searching or retrieval from the images collection are sorted and displayed to users [2].

The CBIR system of classification based is done by predicting the class of the query image based on the classification model formed. The classification model formed is based on existing initial information. Initial information can be obtained from training data or user feedback. The classification model formed is used to predict every image of the collection image [39]. 
In the CBIR system, the user first uploads an image to the system as a query image. The system will perform feature extraction of the query image. To get images that had similarities with the existing query image of the image database, the system compares features owned by the query image with features owned by each image of the feature database. The similarity with the image of the image database with the query image is determined based on the result of the calculation of the distance measurement value used or the predicted model of the classification. The result of calculating the distance measurement value is used to sort the image. The process of sorting the image is done by sorting images from images that have the closest resemblance to the query image to a certain threshold value [40]. Any image that has a measurement value of the distance below or equal to the system-specified threshold value will be expressed as an image that has a close or equal similarity. The image that has similar resemblance to the threshold value will be given to the user as an image retrieval image. The general form of the CBIR system is as illustrated in Fig. 1.

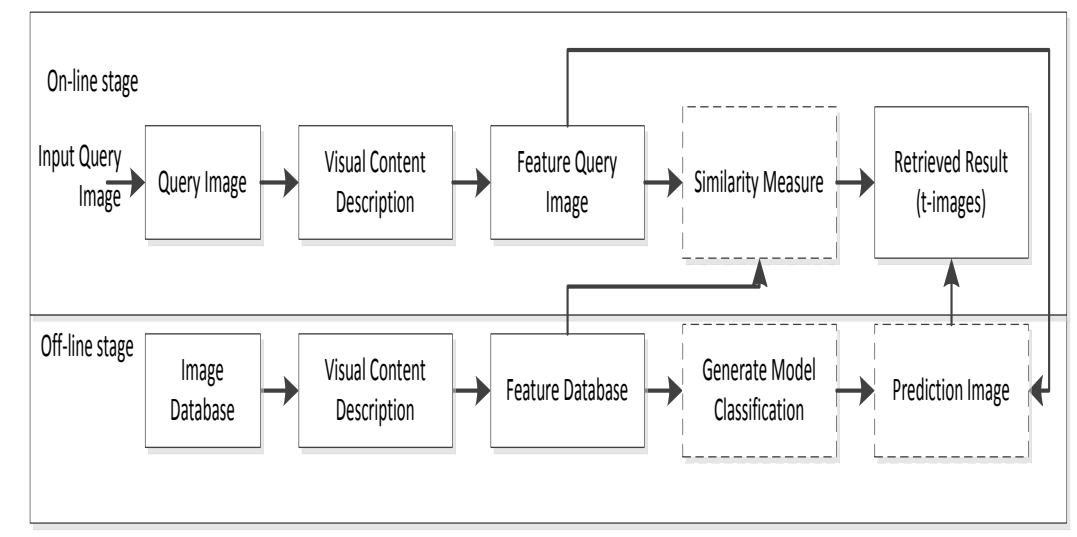

Fig. 1. General form of the CBIR system.

\subsection{HSV Quantization}

HSV (Hue, Saturation, Value) is one type of perceptual color space [36]. HSV has cylindrical coordinates consisting of 3 color channels, namely: hue, saturation, and value. At a true color image can have a number of different colors up to $2^{24}=1,677,7216$ colors, consequently when a true color image is extracted then the features extraction process a true color image will require computation time and large memory [41]. To reduce computation without reducing image quality significantly can be done with Color Quantization [42]. An aim of color quantization is to reduce the number of distinct colors in representing the visual content of an image. A new image produced or obtained from the color quantization process must keep the resemblance to the original image in spite of the change in the number of distinct colors with the original image.

The various HSV Quantization schemes that have been studied include $(8,3,3)[43],(16,4,4),(18,3,3)[44]$, [45], (18,5,3) [46], (19,4,5) [47], and (2,2,10) [41], and others. At HSV Quantization $(19,4,5)$ defines 19 Hue with various step $20(0,20,40, \ldots, 360), 4$ Saturations, step $0.25(0.20,0.40,0.673,0.923)$ and 5 Values, step $0.25(0,0.25,0.5,0.75,1.00)$. At HSV Quantization $(18,5,3)$ defines 18 Hues with various step 20 (350, 10, $30,50, \ldots, 350), 5$ Saturations, step $0.2(0,0.2,0.4,0.6,0.8,1.0)$ and 3 Values, steps $0.25(0,0.5,0.75,1.0)$. At HSV Quantization (8,3,3) defines 8 Hues, step (316-20, 21-40, 41-75, 76-155, 156-190, 191-270, 271-295 and 296-315), 3 Saturations, step (0-0.2, 0.2-0.7 and 0.7-1.0) and 3 Values, step (0-0.2, 0.2-0.7 and 0.7-1.0), and with the same principle done for other schema. So for HSV Quantization $(19,4,5)$ will produce $19 * 4 * 5=$ 380 the number of distinct colors, whereas HSV Quantization $(8,3,3)$ will produce $8 * 3 * 3=72$ the number of distinct colors, and other HSV Quantization schemes. 
One good combination of bins both in terms of performance and computational load is 72 bins (8 bins component $\mathrm{H}, 3$ component bins $\mathrm{S}$ and 3 bins component $\mathrm{V}$ ). The normalization constant of $\mathrm{H}$ is 0 for (315-20), 1 for (20-40), 2 for (40-75), 3 for (75-155), 4 for (155-190), 5 for 190-270], 6 for (270-295), and 7 for (295-315). The normalization constant $S$ is 0 for [0-0.2), 1 for [0.2-0.7], and 2 for (0.7-1) and the normalization constant $V$ is 0 for [0-0.2), 1 for [0.2-0.7], and 2 for (0.7-1) [41], [48]. The HSV Quantization stages are as follows [29]:

a. Convert RGB image into HSV formed

b. Determine the quantization of the color space

c. Determine the normalization of HSV, $\boldsymbol{Q}=9 \boldsymbol{H}+3 \boldsymbol{S}+\boldsymbol{V}$

d. Determine the total amount of each value normalization, Total $_{Q i}=\Sigma\left(Q_{i}\right)$

e. Determine the total value of normalization,

$$
\text { Total }=\Sigma\left(\text { Total }_{Q i}\right)
$$

f. Determine the probability of each quantized color space, $\operatorname{prob}(Q i)=\left(\right.$ Total $\left._{Q i}\right) /($ Total $)$

In this study, each image performs the process of stages a through $\boldsymbol{f}$ both of the images collection and a query image. At the off-line feature extraction, the result of step processes $\boldsymbol{a}$ until $\boldsymbol{f}$ will be stored in the feature database. So the color feature extraction of each image will be represented in the probability values of color space quantization. While the online feature extraction is also doing step process $\boldsymbol{a}$ until $\boldsymbol{f}$ that using to calculate the similarity measure between the query image and each of the images collection.

\subsection{Support Vector Machine (SVM)}

SVM is a supervised learning method that generates a function or model based on mapping the input-output from a set of the training data that has been labeled [38], [49]. SVM uses hypothetical space in the form of linear functions of a feature space of high-dimensional features, training based on learning algorithms oriented to learning theory by implementing biases derived from learning theory [50]. The learning process of SVM is aimed to get the hypothesis of the best separate field. The purpose of SVM is to generate a model (based on training data) that predicts the target value of the test data provided. The best separate field is obtained by finding the empirical risk (average error on the training data) minimal and also having excellent generalizations.

The feature space in practice usually has a higher input vector dimension (input space). This produces computing in the feature space becomes very large. The larger feature space caused by computing feature space can have an unlimited number of features and it is difficult to know the exact transformation function [51]. To solve this problem, SVM uses the "kernel trick". A function can be converted into a specific kernel function if it satisfies with the Mercer's theorem. There are several commonly used kernel functions including linear kernels, polynomial kernels, radial base functions (RBF), and sigmoid kernels [49].

The basic theory of SVM has actually been developed since 1960. But SVM is becoming a very popular and rapidly growing technique after SVM was introduced by Vapnik, Boser, and Guyon since 1992 [52]. Currently, Classification, Clustering, and Regression fields have used Support Vector Machines (SVMs) extensively. The term SVM is usually used to describe classification and regression. Classification is done by using Support Vector Classification (SVC) methods while for regression using Support Vector Regression (SVR) [50]. Some applications that have used SVM include bioinformatics, text mining, face recognition, and image processing.

The key idea of SVM is to study the optimum hyperplane that separates the training examples with the maximal margin [50]. A linear SVM finds an optimal hyperplane $f(x)=w^{T} x+b$. The hyperplane SVM is a 
straight line or a horizontal field that separates classes. The hyperplane can be written as a set of feature sets $\mathrm{x}$ which satisfies the equation $\mathrm{w}^{\mathrm{T}} \mathrm{x}+\mathrm{b}=0$. All vectors on one side of the SVM hyperplane are labeled with +1 while on the other side are labeled with -1 . The training instances closest to the hyperplane are called as support vectors [53]. To achieve the separation of the two best classes the function $f(x)=w^{T} x+b$ is obtained by finding the empirical risk minimum and the largest margin $\mathrm{w}$ based on properly classified training vectors. So the best hyperplane is the hyperplane located halfway between two sets of objects of two classes.

\subsection{Support Vector Machine Relevance Feedback (SVM RF)}

Initially, Relevance Feedback (RF) technique was used in document retrieval, i.e. at SMART [54]. The RF techniques used in CBIR began in the early 1990s [27]. As for that causes the need for user feedback is because of the different needs of each user (Kurito and Kato, 1993) and the different needs at different times. So as to facilitate the needs of various users, various times and various situations with both the required feedback from users.

In Coex, IJ, et al. [55] states that the user to search the desired image has two ways, that is oriented to the category search and target search. Therefore, in developing RF technique in CBIR, it is necessary to pay attention to user orientation using a system. In general, the RF techniques in the CBIR systems have four components: user interface, offline learning, data storage, and online learning [56]. Thomee and Lew [56] stated that the main challenge in developing the CBIR system with RF techniques is to bridge the semantic gap by improving conceptual or user perception techniques, solving dimensional problems to select optimal features, and reducing the number of iterations to achieve convergence.

The semantic gap in the CBIR system can be reduced efficiently using RF [26], [57]. Patil [58] also revealed that to define $\mathrm{RF}$ technique and scheme needs to take into account certain assumptions and criteria. Saju, A., et al. [20] revealed that one way to raise the accuracy of the CBIR system can be done by using RF. But Saju and his team revealed that the biggest challenge to the CBIR system with RF techniques is the number of iterations and execution times required by the system.

$\mathrm{RF}$ is an online process that tries to learn the user's interest based on the feedback given. User interest will lead to recurrence of the feedback process in reducing the semantic gap between the represented query (low-level features) and the user's thought (semantic features) [39]. RF will provide better the CBIR system search performance based on continuous system learning of user feedback [29].

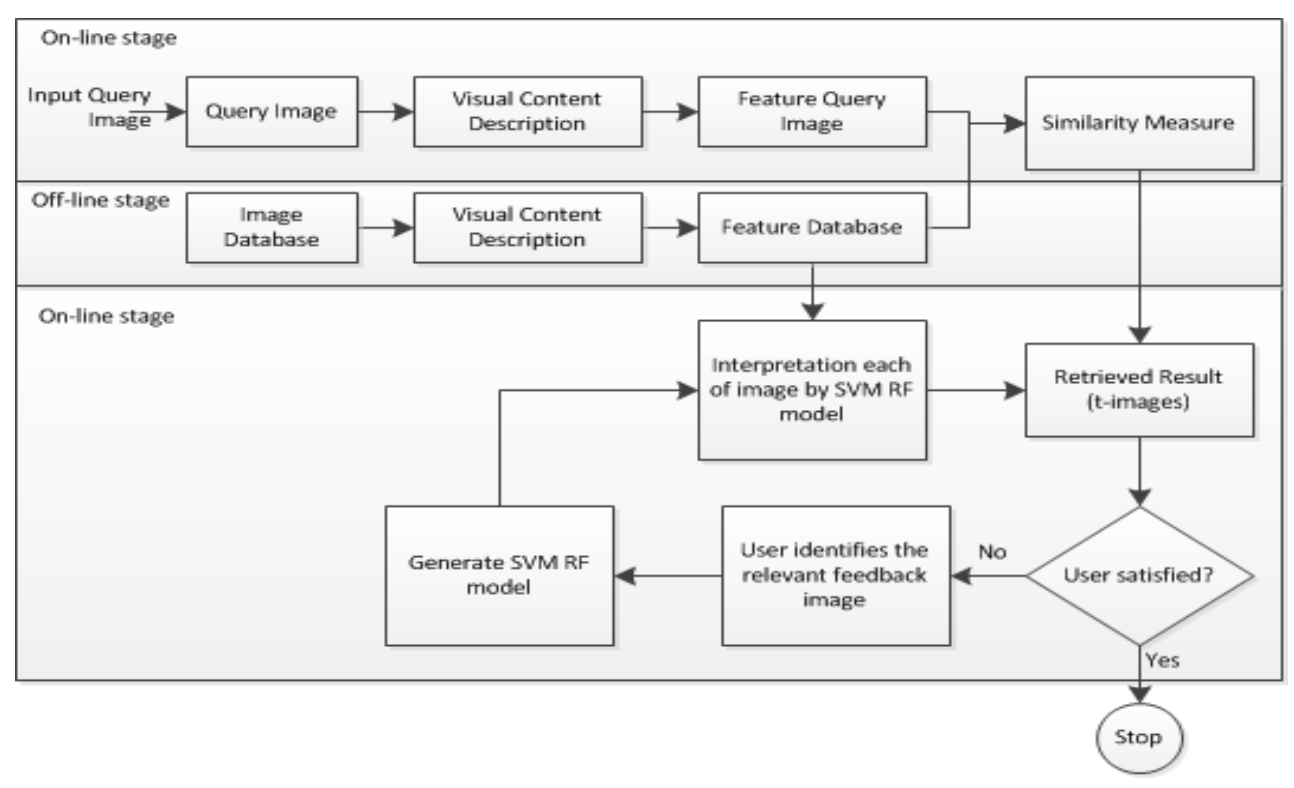

Fig. 2. General form of the SVM RF in the CBIR system. 
In Hoi, $\mathrm{SCH}$, et al. [59] revealed that $\mathrm{RF}$ is a powerful tool to improve the performance of the CBIR system. Hoi also revealed that the development of machine learning techniques also affected the development of RF techniques on CBIR. Some of the machine learning methods used in RF techniques included Bayesian learning, decision tree, boosting techniques, discriminant analysis, dimension reduction, ensemble learning, SOM, EM algorithms, and SVM [31]. The general shape of SVM RF in the CBIR system in this study as shown in Fig. 2. Stages SVM RF is as follows:

a. Input the query image

b. Extract feature low-level query image with feature extraction HSV Quantization

c. Compare every imagery in the image database

d. The system provides early image retrieval results based on the query image.

e. The user asses the results provided by the system, it is appropriate or not. If yes go to step $\boldsymbol{k}$, otherwise go to step $\boldsymbol{f}$.

f. Select the relevant images as a feedback. For each feedback images given as label +1 , while the other images are labeled -1 . Then the system forms the target vector +1 or -1 .

g. The vector feature form corresponds to users' feedback with the vector feature element corresponding to the image vector feature that has been stored in the feature database.

h. Generate SVM RF model based on users' feedback.

i. Determine the classification of each image based on prediction SVM RF model.

j. Show the image retrieval feedback results based on SVM RF prediction. If it is in accordance with the wishes of the user go to step $\boldsymbol{k}$, if not repeat to step $\boldsymbol{f}$.

k. Done.

For the establishment of an SVM RF model based on user feedback at stage $\boldsymbol{h}$ it is necessary to balance the number of user-selected feedback images. For the amount of unbalanced feedback image is done by generating set of new image feedback based on existing image features. For the number of feedback images smaller than the number of unselected images, the feedback image will be generated to form a new feedback image. Conversely, the number of image feedback larger than the number of unselected images, the negative image training data will be generated based on the negative image training data feature. The process of forming a new image is done to form a balanced training data between relevant images with irrelevant images. Let $\boldsymbol{S r}=\left\{\boldsymbol{i m}_{1}, \boldsymbol{i m}_{2}, \ldots, \boldsymbol{i} \boldsymbol{m}_{r}\right\}$ is a set of relevant feedback images and $\boldsymbol{r}$ is the number of relevant feedback images. Let $\boldsymbol{S n}=\left\{\boldsymbol{i m}_{1}, \boldsymbol{i m}_{2}, \ldots, \boldsymbol{i} \boldsymbol{m}_{\boldsymbol{n}}\right\}$ is a collection of irrelevant images and $\boldsymbol{r}$ is the

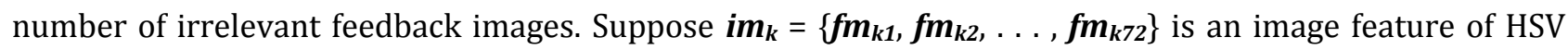
Quantization of the image-k. For $\boldsymbol{r}<\boldsymbol{n}$, it will form a number of $(\boldsymbol{n}-\boldsymbol{r})$ new image based on image feature of HSV Quantization from the elements of $\boldsymbol{S} \boldsymbol{r}$. Whereas if $\boldsymbol{r}>\boldsymbol{n}$, it will form a number of $(\boldsymbol{r}-\boldsymbol{n})$ new image based on image feature of HSV Quantization from the elements of $\boldsymbol{S n}$. To form a new image, for example im $_{\text {new }}$, is done based on the framing of the randomly selected image feature of HSV Quantization. Suppose to

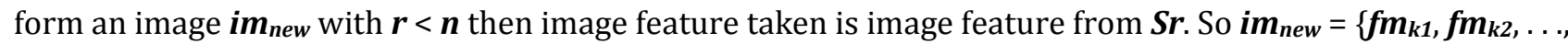
$\left.\boldsymbol{f m}_{\boldsymbol{r 7 2}}\right\}$ where $\boldsymbol{k}$ is any random element $\boldsymbol{r}$. For $\boldsymbol{f m}_{21}$ means that the $1^{\text {st }}$ image feature is taken from the $1^{\text {st }}$ feature of the $2^{\text {nd }}$ feedback image and for $\boldsymbol{f m}_{32}$ means that the $2^{\text {nd }}$ feature is taken from the $2^{\text {nd }}$ feature of the $3^{\text {rd }}$ feedback image. Likewise for $\boldsymbol{f} \boldsymbol{m}_{r 72}$ means that the $72^{\text {nd }}$ feature is taken from the $72^{\text {nd }}$ feature of the $\mathrm{r}^{\text {th }}$ feedback image.

\subsection{Wang Dataset}

Wang Dataset has 1000 images with file type is jpg. Each image has a varying capacity, between $7 \mathrm{~KB}$ to $55 \mathrm{~KB}$, with dimensions of $256 \times 384 \mathrm{px}$ or $384 \times 256 \mathrm{px}$. Wang dataset has 10 categories, each category being Africans, Beaches, Building, Bus, Dinosaur, Elephant, Flower, Horses, Mountain, and Food. Each category of Wang dataset has 100 images. One example of the image of each category is as shown in Fig. 3. 

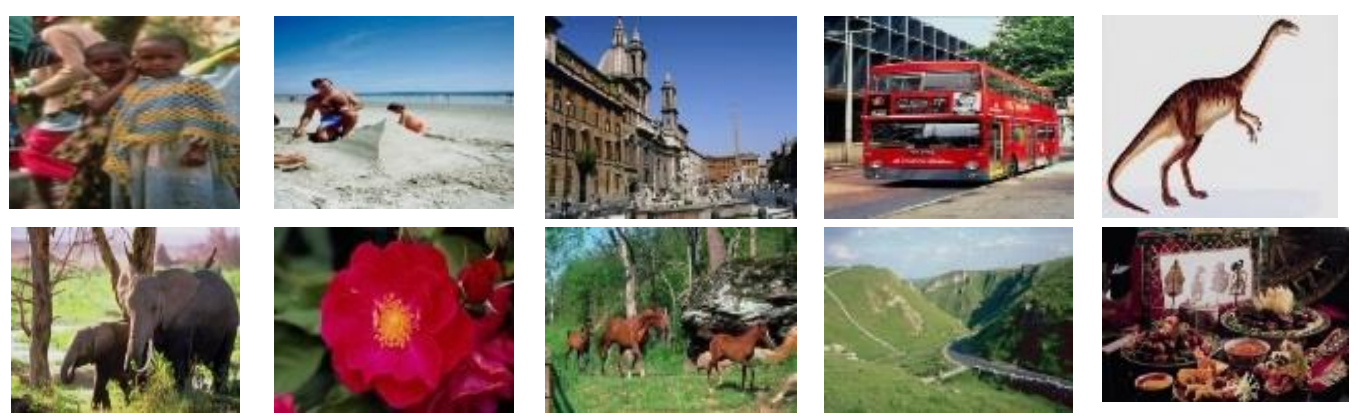

Fig. 3. The example of wang dataset.

\section{Experiment Results and Discussions}

In this research uses the SVM RF technique. The proposed technique has two stages. The first stage is the image retrieval process based on Jeffrey Divergence with threshold 0.15. The second stage is the image retrieval process based on SVM RF model prediction. In the SVM relevance feedback technique, the user gives feedback to result of the CBIR system as a relevant image by choosing images that match to the image query. Furthermore, user feedback images are used to form the SVM RF model. The SVM RF model is used to predict the relevant images with the query image.

To observe the performance of SVM relevance feedback in CBIR, this research conducts an experiment to the Wang dataset. First of all, the system performed feature extraction to the Wang dataset. The feature extraction used to get the features of each image in the dataset. The extracted image features are stored in the feature database. The feature extraction that used in this study implements HSV Quantization $(8 \times 3 \times 3)$ so that each image has 72 features, as described in Section 3. In the image search phase, the same process is performed on the query image. The system will compare 72 features of the query image that extracted to each image features that stored in the feature database. The distance measurement uses Jeffrey Divergence done to determine a match between the query image and the image in the database. The CBIR system displays the result of the distance measurement to the user that will be used to the next stage.

In this study, the performance of the SVM RF on Wang dataset is observed using the RBF kernel and linear kernel. The result of the testing of the SVM RF using the RBF kernel is described in section 3.1, the result of the testing of the SVM RF using linear kernel is described in section 3.2, while the comparison of the performance of the SVM RF to the state-of-the-art method is explained in section 3.3.

\subsection{Experiment Result of SVM RF with RBF Kernel}

Testing on the Wang dataset is done by taking the existing imagery on Wang dataset as an image query. Tests conducted in this study used 10-fold cross validation for each category of its image. So for 1000 images Wang existing datasets, 100 images taken for the test images with each of its categories is 10 images. The performance of SVM relevance feedback technique on the Wang dataset is observed in terms of precision, recall, F-measure, and accuracy. The performance of the SVM relevance feedback technique is also concerned with the selection of kernel types used, the determination of the constant value $(\mathrm{C}, \mathrm{G})$ for the RBF kernel, and the determination of the constant value $\mathrm{C}$ for the linear kernel.

The determination of the constant value $(\mathrm{C}, \mathrm{G})$ is done by $2^{\mathrm{k}}$ with $\mathrm{k}$ is a real number ranging from 0 to a large value [47]. In this research, the selection of the constant value $(C, G)$ is started from the largest value to the smallest value, then observing the performance of the SVM RF. The best value of $\mathrm{k}$ obtained in each experiment is used as a reference to replacing the new $\mathrm{k}$ value. As for the result of SVM RF with RBF kernel for various values of constants (C, G) on Wang dataset each feedback image selection with the $2^{\text {nd }}$ feedback is as stated in Table 1. 
Table 1. Precision, Recall, and F-Measure the SVM RF with RBF Kernel by Various of C and G

\begin{tabular}{|c|c|c|c|c|c|c|c|c|c|}
\hline \multirow{2}{*}{ Coef. ( C, G) } & \multicolumn{3}{|c|}{ Base line } & \multicolumn{3}{|c|}{ FB-1 } & \multicolumn{3}{|c|}{ FB-2 } \\
\hline & Precision & Recall & F-Measure & Precision & Recall & F-Measure & Precision & Recall & F-Measure \\
\hline $2^{15.00}: 2^{15.00}$ & 0.6592 & 0.2949 & 0.4075 & 0.5519 & 0.6140 & 0.5813 & 0.6464 & 0.7474 & 0.6932 \\
\hline $2^{10.00}: 2^{15.00}$ & 0.6592 & 0.2949 & 0.4075 & 0.5520 & 0.6147 & 0.5817 & 0.6475 & 0.7479 & 0.6941 \\
\hline $2^{4.00}: 2^{4.00}$ & 0.6592 & 0.2949 & 0.4075 & 0.5621 & 0.6079 & 0.5841 & 0.6529 & 0.7377 & 0.6927 \\
\hline $2^{3.00}: 2^{3.00}$ & 0.6592 & 0.2949 & 0.4075 & 0.4773 & 0.6770 & 0.5599 & 0.6485 & 0.7960 & 0.7147 \\
\hline $2^{2.00}: 2^{2.00}$ & 0.6592 & 0.2949 & 0.4075 & 0.4018 & 0.7183 & 0.5154 & 0.6746 & 0.7598 & 0.7146 \\
\hline $2^{2.50}: 2^{2.50}$ & 0.6592 & 0.2949 & 0.4075 & 0.4463 & 0.7040 & 0.5463 & 0.6721 & 0.7922 & 0.7272 \\
\hline $2^{2.70}: 2^{2.70}$ & 0.6592 & 0.2949 & 0.4075 & 0.4463 & 0.7040 & 0.5463 & 0.6721 & 0.7922 & 0.7272 \\
\hline $2^{2.725: 2^{2.725}}$ & 0.6592 & 0.2949 & 0.4075 & 0.4585 & 0.6959 & 0.5528 & 0.6791 & 0.8006 & 0.7348 \\
\hline $2^{2.75}: 2^{2.75}$ & 0.6592 & 0.2949 & 0.4075 & 0.4604 & 0.6951 & 0.5539 & 0.6769 & 0.7994 & 0.7331 \\
\hline $2^{2.80}: 2^{2.80}$ & 0.6592 & 0.2949 & 0.4075 & 0.4637 & 0.6920 & 0.5553 & 0.6667 & 0.7999 & 0.7273 \\
\hline
\end{tabular}

Based on the results of the research stated in Table 1, it is obtained that the SVM RF provides excellent performance for each given constant value. This shows that the SVM RF provides better performance when compared with no feedback, especially recall and F-measure values. In the first feedback, the SVM RF generally does not increase the precision value but increases the recall and F-measure values. While on the $2^{\text {nd }}$ feedback some the SVM RF can increase the value of precision, recall, and F-measure. Degradation of precision value in the first feedback and the $2^{\text {nd }}$ feedback is caused by increasing the number of images taken based on results of prediction of the SVM RF model but they are not comparable with the number of relevant images retrieved. But, when compared with the relevant image on the baseline at the first and the second feedback has the same number or more relevant images retrieved.

The SVM RF with $R B F$ kernel to Wang dataset has the best performance on the constant value $(\boldsymbol{C}, \boldsymbol{G})=$ $\left(2^{2.725}, 2^{2.725}\right)$. On the $2^{\text {nd }}$ feedback with $(\boldsymbol{C}, \boldsymbol{G})=\left(2^{2.725}, 2^{2.725}\right)$ can improve the performance of precision by $3.02 \%$ i.e. baseline of HSV Quantization with distance measurement Jeffrey Divergence by threshold 0.15 that has the precision value amount $65.92 \%$ while the SVM RF achieves $67.91 \%$. For the recall value, the SVM RF increases the recall value amount $171.48 \%$, i.e. the baseline of HSV Quantization has the recall value $29.49 \%$ while the SVM RF has $80.06 \%$. For the F-measure value of the SVM RF also increased by $80.34 \%$, i.e. the baseline only reach $40.75 \%$ while with the SVM RF can reach $73.48 \%$.

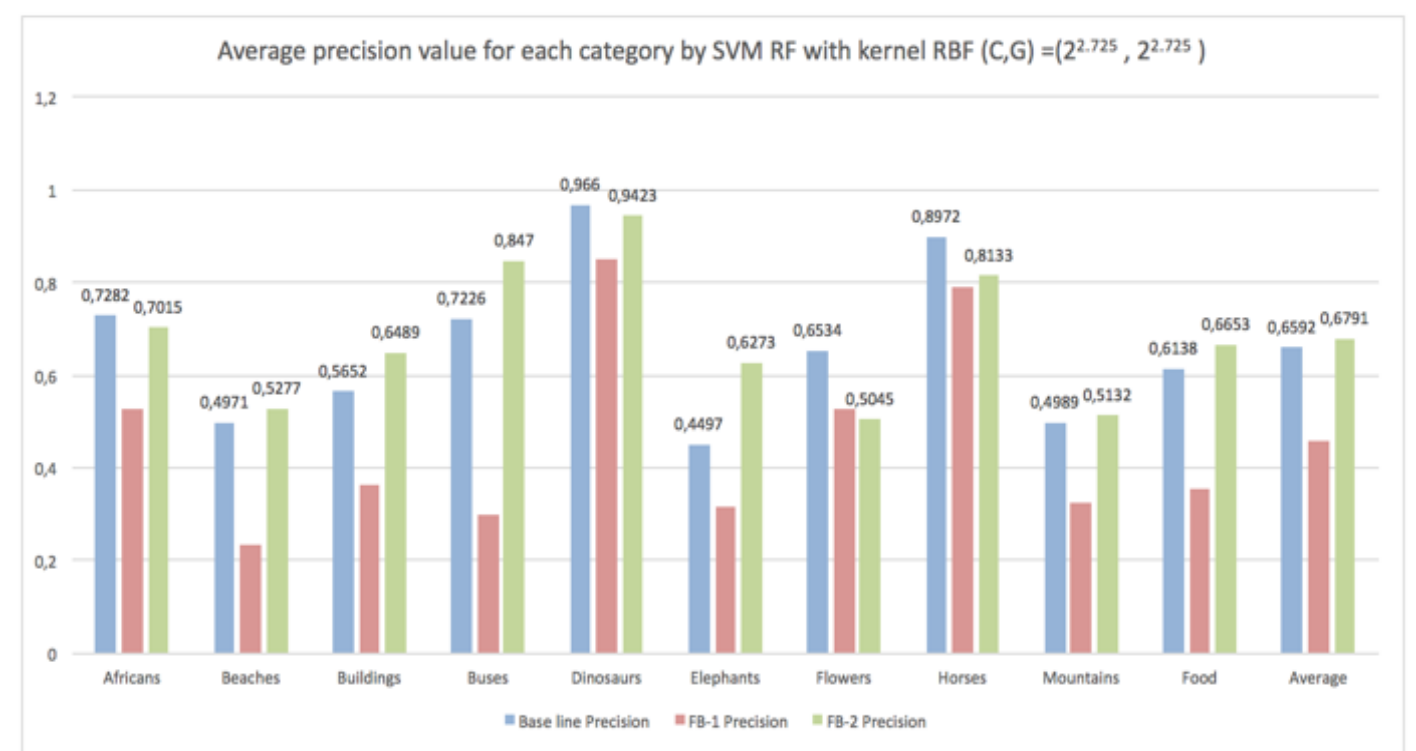

Fig. 4. The average precision value of the SVM RF with RBF kernel with $(\boldsymbol{C}, \boldsymbol{G})=\left(2^{2.275}, 2^{2.275}\right)$ each category.

Results of testing the SVM RF with $R B F$ kernel with a constant value $(\boldsymbol{C}, \boldsymbol{G})=\left(2^{2.725}, 2^{2.725}\right)$ are also 
observed on each category of the Wang dataset. The average precision values of the SVM RF on the first feedback are always lower than the average precision value of base line. But the average precision value of the SVM RF on the second feedback generally have a better average precision value. The SVM RF on the second feedback obtains the best average precision value in 6 categories, namely Beaches, Buildings, Buses, Elephants, Mountains, and Food category. The best average precision value of the SVM RF on the second feedback is the Elephants category, which can increase the average precision value amount $39.50 \%$. The graphical representation of the average precision value of the SVM RF with $R B F$ kernel with a constant value $(\boldsymbol{C}, \boldsymbol{G})=\left(2^{2.725}, 2^{2.725}\right)$ is as represented in Fig. 4 .

The result of testing of the RF SVM with $R B F$ kernel with $(\boldsymbol{C}, \boldsymbol{G})=\left(2^{2.725}, 2^{2.725}\right)$ in each category Wang dataset always gives better results for all its categories. At the first feedback gives excellent recall value. In the first feedback of the SVM RF can increase the average recall value amount $81.95 \%$, i.e. in the Beaches category, and then followed by Mountain category amount 78.81\%.

When compared to the recall results of the first feedback with the second feedback, the average recall value of the SVM RF the second feedback can only increase the average recall value amount $15.04 \%$. Otherwise, when compared to the average recall value of the SVM RF on the second to base line, the SVM RF can increase the average recall value amount $171.48 \%$. The largest average recall value of the SVM RF on the second feedback is category Dinosaurs, i.e. $99.89 \%$, and then followed by Horses category, which is 99.11\%. But the SVM RF on the second feedback gives the best performance in the Beaches category, which can increase the average recall value amount $678.09 \%$. This shows that SVM RF can represent the feature of the feedback image vector as a specific category vector feature. The graphical representation of the average recall value of the RF SVM with $R B F$ kernel with $(\boldsymbol{C}, \boldsymbol{G})=\left(2^{2.725}, 2^{2.725}\right)$ is as shown in Fig. 5.

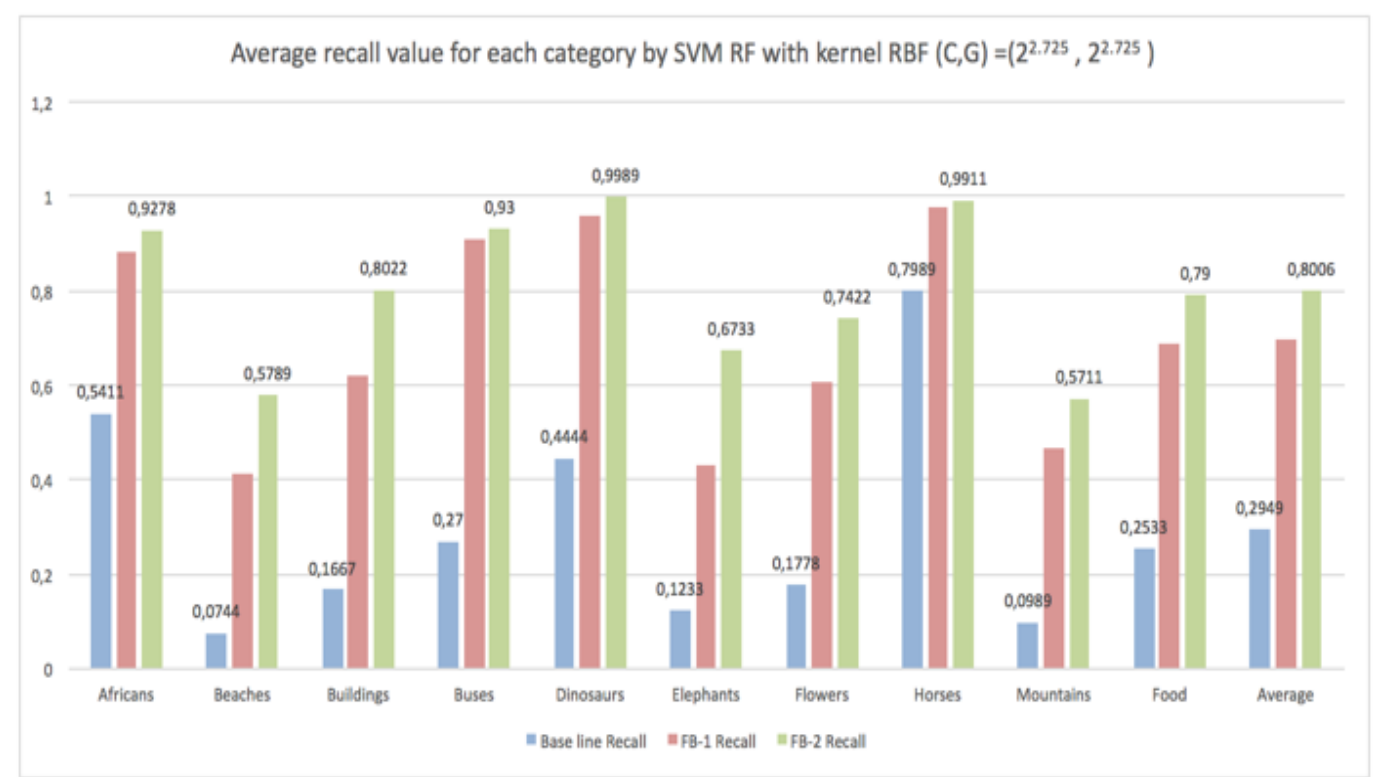

Fig. 5. The average recall value of the SVM RF with RBF kernel with $(\boldsymbol{C}, \boldsymbol{G})=\left(2^{2.275}, 2^{2.275}\right)$ each category.

The average $F$-measure value of the SVM RF with RBF kernel with the constant value $(\boldsymbol{C}, \boldsymbol{G})=\left(2^{2.725}, 2^{2.725}\right)$ in each category also always gives better value. In the first feedback of the SVM RF has provided an increase the F-Measure value amount $35.65 \%$. The best performance of the F-measure value is obtained in the Mountains category, which increases the F-measure value amount $131.50 \%$. The SVM RF on the second feedback repairs the first model of the SVM RF so obtained an increase of F-Measure value amount 32.94\%. This suggests that improvements to the SVM RF model will achieve the better performance. According to the experiment result, the second of the SVM RF model yields the better performance compared to the first 
SVM RF models.

The best average F-measure value of the SVM RF on the second feedback is Dinosaurs category i.e. 96.98\%, followed by Horses category i.e. 89.34\%. While the third position is the Buses category, which is $88.66 \%$. The graphical representation of the average F-measure of the SVM RF with $R B F$ kernel with $(\boldsymbol{C}, \boldsymbol{G})=\left(2^{2.725}\right.$, $2^{2.725}$ ) is as shown in Fig. 6.

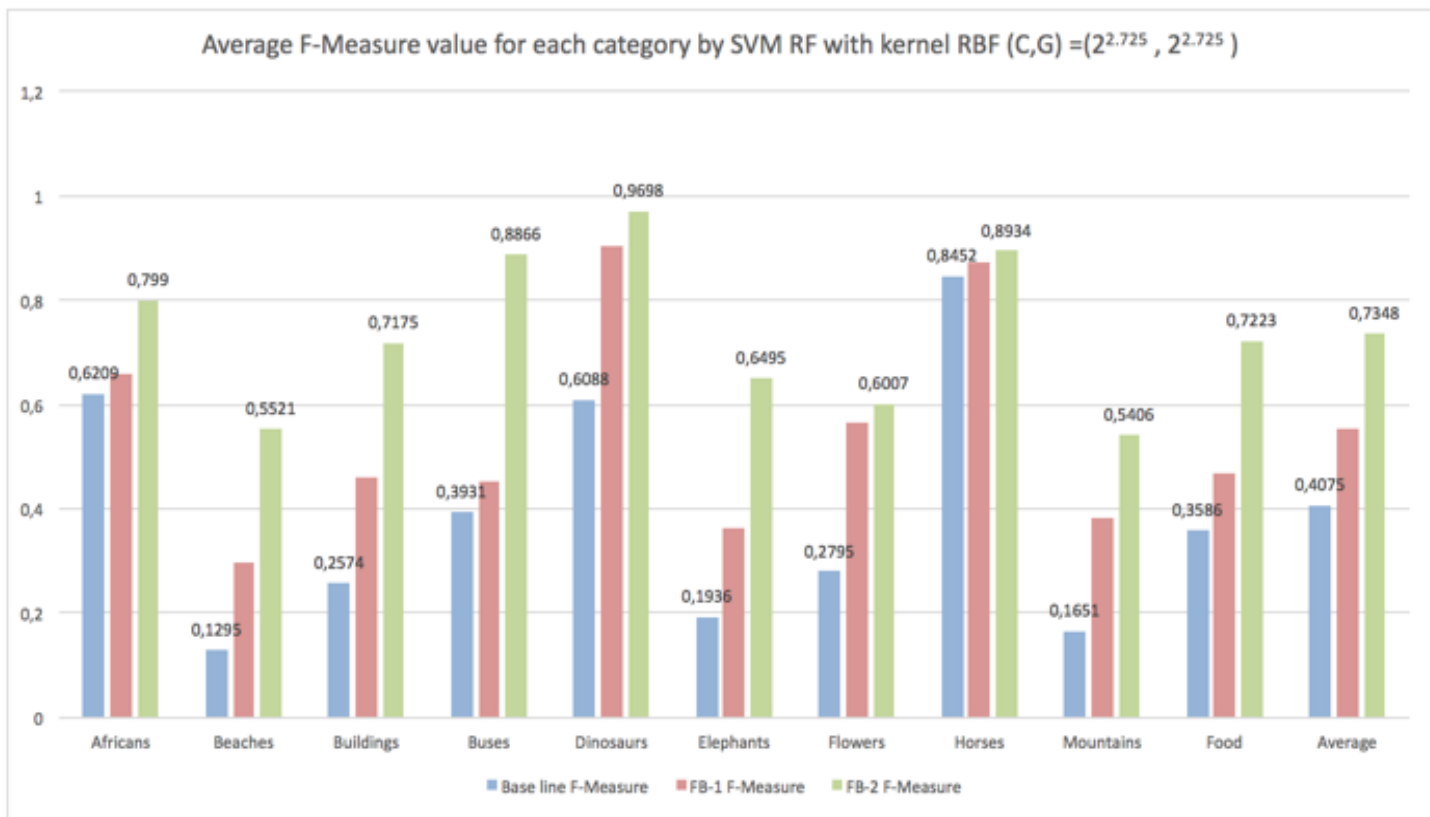

Fig. 6. The avergae F-measure value of the SVM RF with RBF kernel with $(\boldsymbol{C}, \boldsymbol{G})=\left(2^{2.275}, 2^{2.275}\right)$ each category.

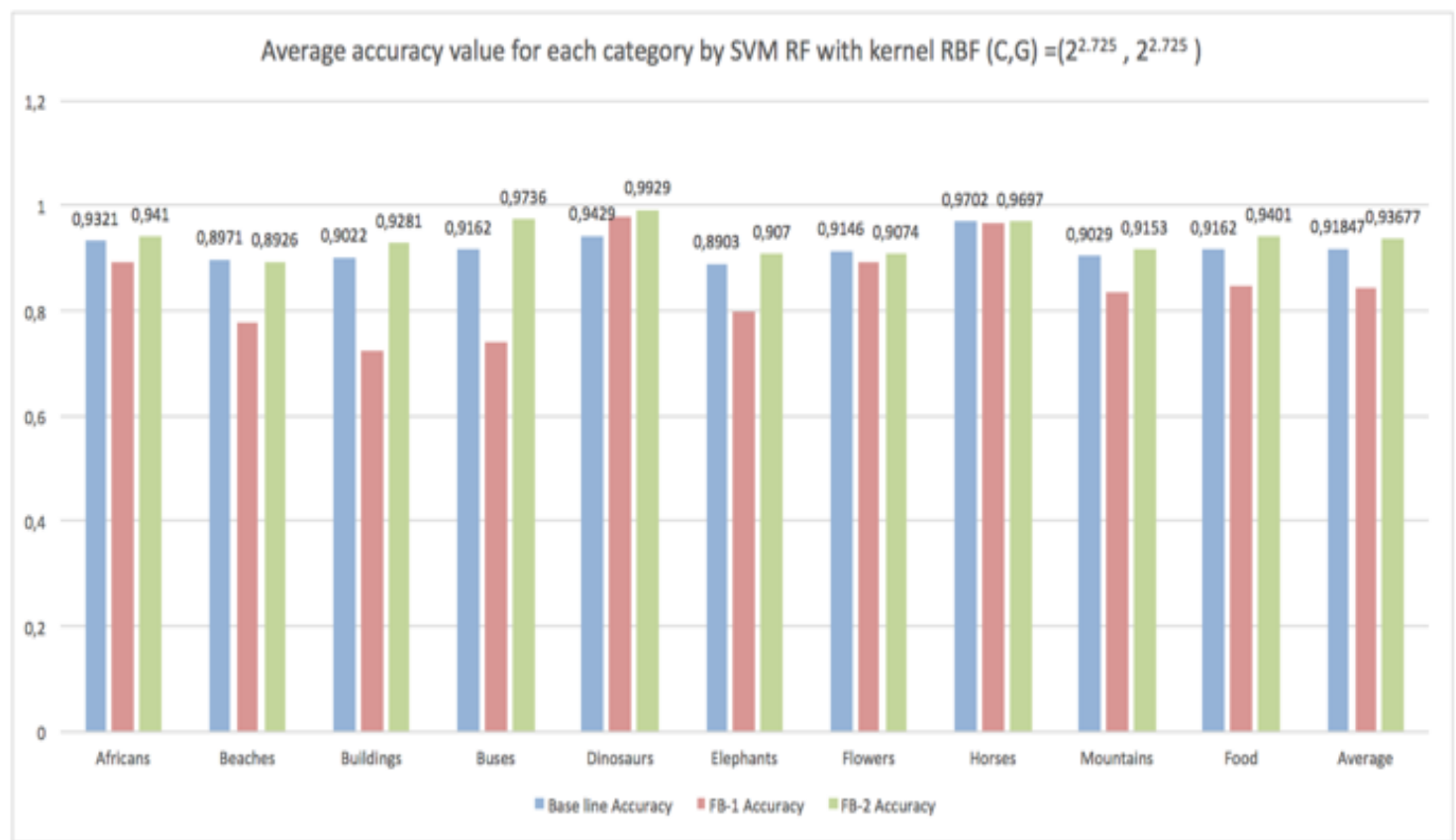

Fig. 7. The average accurracy value of the SVM RF with RBF with $(\boldsymbol{C}, \boldsymbol{G})=\left(2^{2.275}, 2^{2.275}\right)$ each category.

Results of testing of the SVM RF with $R B F$ kernel with a constant value $(\boldsymbol{C}, \boldsymbol{G})=\left(2^{2.725}, 2^{2.725}\right)$ in each category Wang dataset based on accuracy is as stated in Fig. 7. In the first feedback of the SVM RF is generally lower. The decrease in the average accuracy value of the SVM RF is due to the increasing number of images retrieved except in the Dinosaurs category which rose $3.47 \%$. While the SVM RF with RBF kernel 
on the second feedback increased the average accuracy value in 7 categories, namely Africans, Buildings, Buses, Dinosaurs, Elephants, Mountains, and Foods. The best average accuracy value was achieved on the Dinosaurs category i.e. $99.29 \%$, followed by the Buses category i.e. $97.36 \%$. The third best accuracy is the Horses category i.e. $96.97 \%$. Based on the result of the average accuracy value of the SVM RF with RBF kernel can be concluded that SVM RF also can improve the accuracy value of the CBIR system.

\subsection{Experiment Result of SVM RF with Linear Kernel}

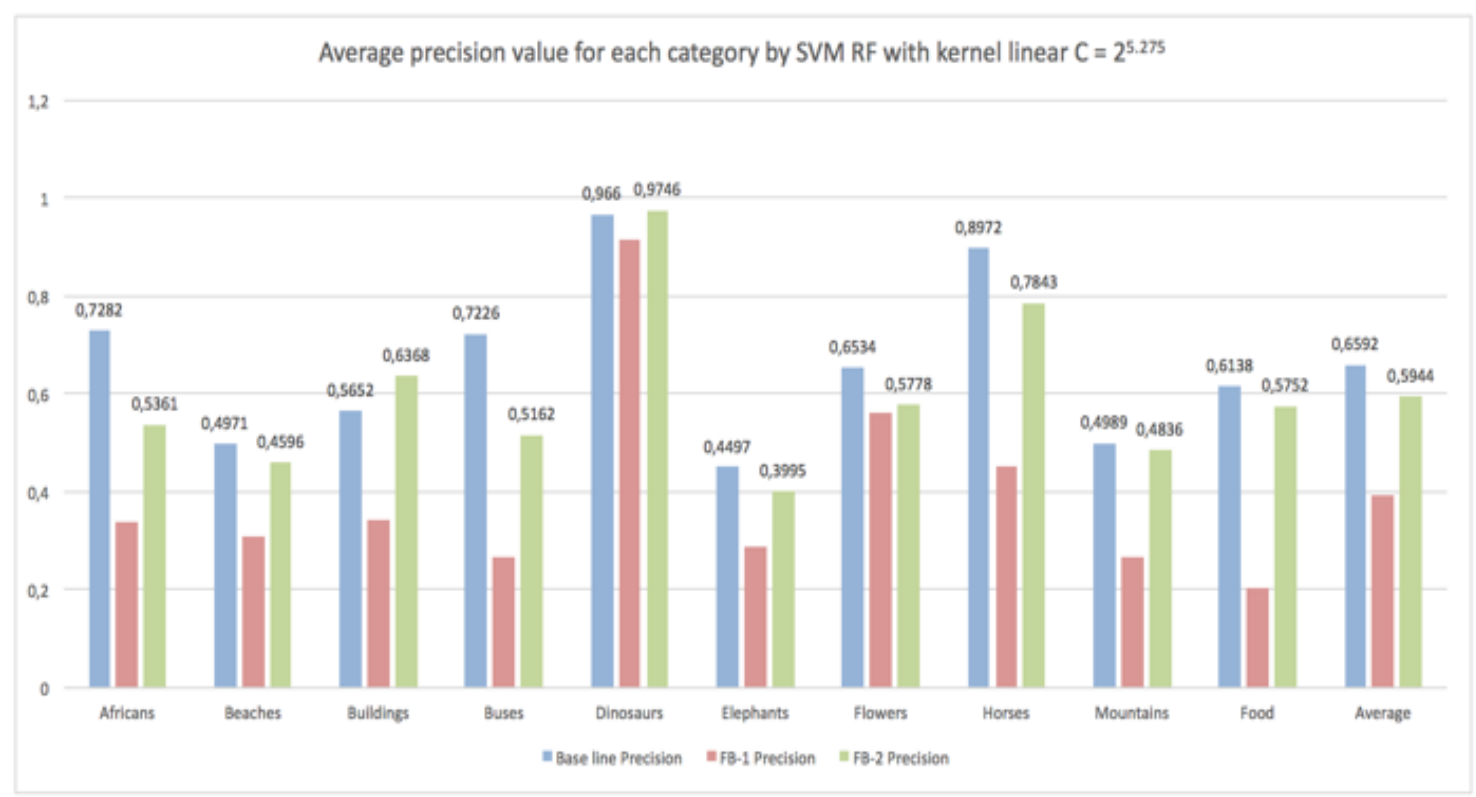

Fig. 8. The average precision value of the SVM RF with linear kernel with $\boldsymbol{C}=2^{5.275}$ each category.

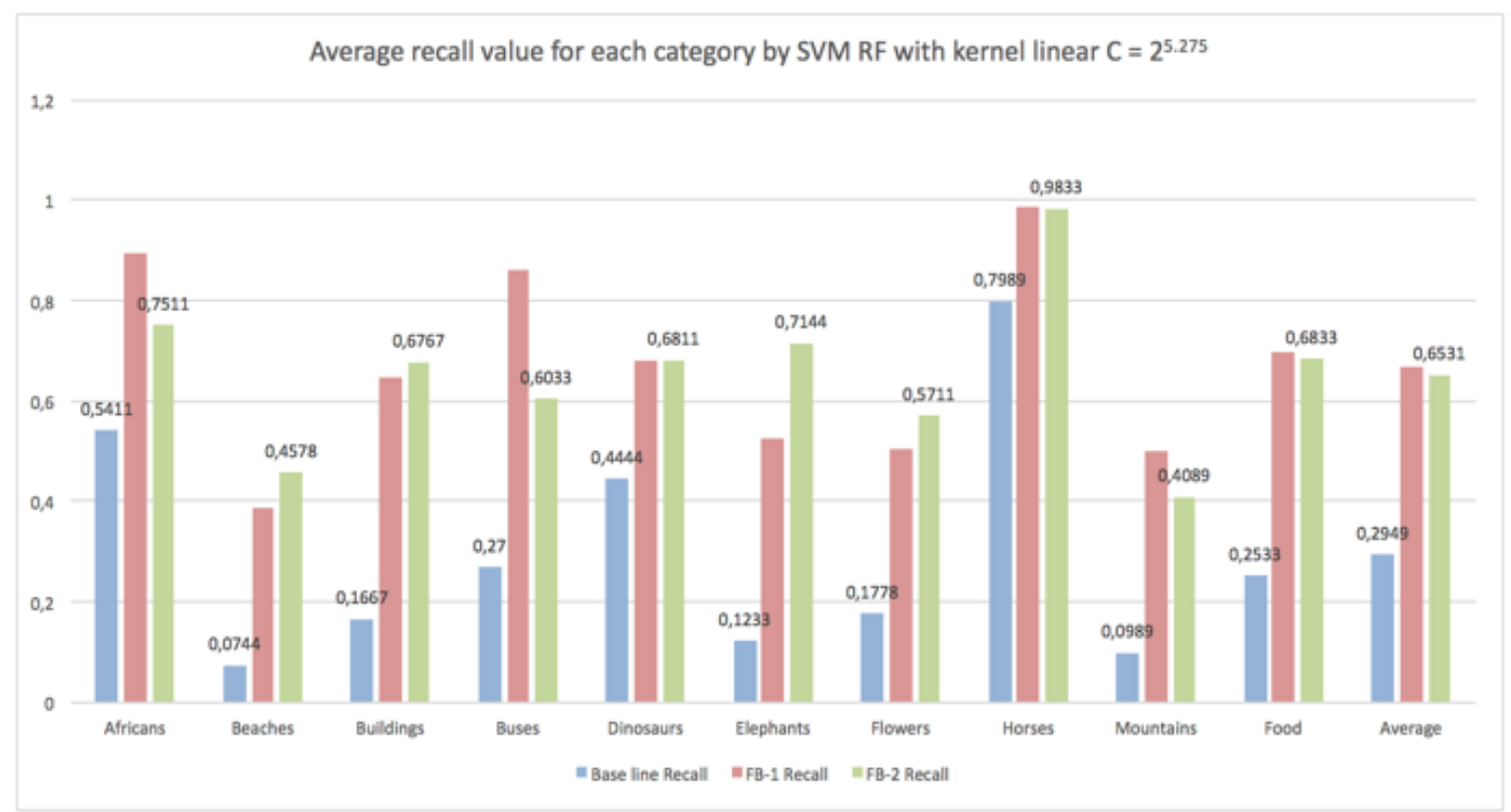

Fig. 9. The average recall value of the SVM RF with linear kernel with $\boldsymbol{C}=2^{5.275}$ each category.

In the same way with the testing of the SVM RF with RBF kernel, in the SVM RF with the linear kernel is also done to find the best value of the constant $C$, which is $C=2^{k}$ with $\boldsymbol{k}$ is a real number value from 0 to a large value. Based on the result of testing of the SVM RF with the linear kernel of constant $\boldsymbol{k}$ on Wang dataset each feedback image selection and using the $2^{\text {nd }}$ feedback, obtained the best value of $\boldsymbol{C}$ at $\boldsymbol{k}=5.275$. 
In the SVM RF with the linear kernel with constant value $\boldsymbol{C}=2^{5.275}$ also gives the average precision value at the first feedback is smaller than the average precision value of its base line. But on the second feedback of the SVM RF with the linear kernel can increase the average precision value in the category of Buildings amount $12.67 \%$ and in the category of Dinosaurs amount $0.89 \%$. The graphical representation of the average precision value of the SVM RF with the linear kernel with $\boldsymbol{C}=2^{5,275}$ is as shown in Fig. 8 .

The average recall value of the SVM RF with the linear kernel with a constant value of $\boldsymbol{C}=2^{5.275}$ in each category gives larger the average recall value. In the second feedback, the average recall value of the SVM RF with the linear kernel provides the average recall value that smaller than the first feedback on four categories i.e. Africans, Buses, Horses, and Mountains. The graphical representation of the average recall value of the SVM RF with the linear kernel is as shown in Fig. 9.

Based on the average precision value and the average recall value of the SVM RF with the linear kernel with constant $\boldsymbol{C}=2^{5.275}$ which does not always rise in the first and the second feedback will affect the average F-measure value. The average F-measure of the SVM RF with the linear kernel value with the constant $\boldsymbol{C}=2^{5.275}$ gives the best $F$-measure value on the second feedback. At the first feedback of the SVM RF with the linear kernel achieves the better F-Measure value in seven categories, namely Beaches, Buildings, Buses, Dinosaurs, Elephants, Flowers, and Mountains. While the average F-measure value on the second feedback of the SVM RF with the linear kernel rises for each category. The best average F-measure value is the Horses category which is $87.26 \%$. The second best F-Measure value is Dinosaurs category, which is $80.18 \%$. While the third best F-measure value is Building category, which is $65.61 \%$. The graphical representation of the average $F$-measure value of the SVM RF with the linear kernel with the constant value $\boldsymbol{C}=2^{5.275}$ is as shown in Fig. 10.

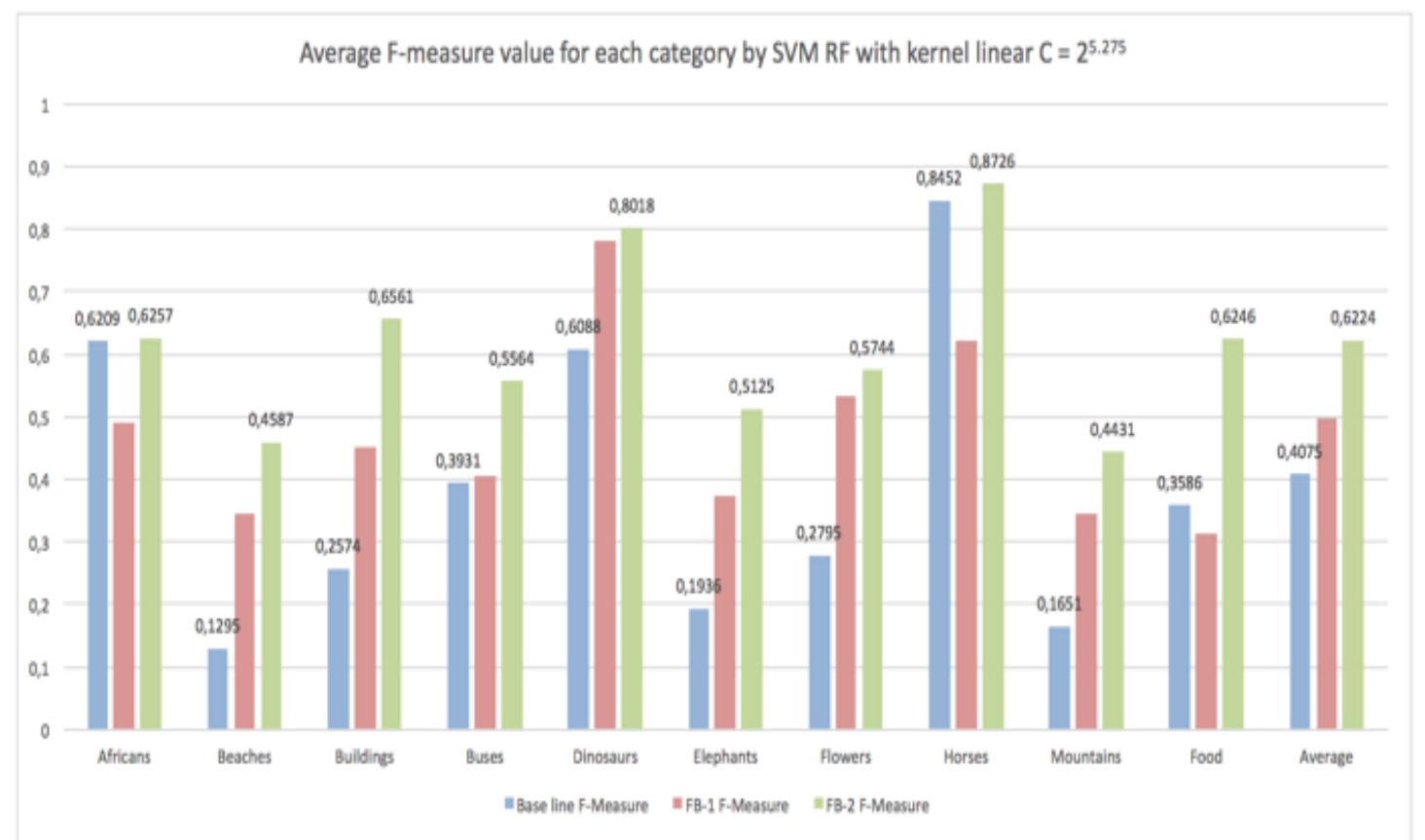

Fig. 10. The average F-measure value of the SVM RF with linear kernel with $\boldsymbol{C}=2^{5.275}$ each category.

Based on the average precision value, the average recall value, and the average F-Measure value that obtained from the experimental result on both of the SVM RF with the RBF kernel and the SVM RF with the linear kernel obtained that the SVM RF with the RBF kernel has the best performance. Therefore, it can be concluded that for the SVM RF on Wang dataset has the best performance with the RBF kernel with constants $(\boldsymbol{C}, \boldsymbol{G})=\left(2^{2.725}, 2^{2.725}\right)$. 


\subsection{Comparison of the Performance of the SVM RF with the State-of-the-Art Method}

The experimental result of the SVM RF with $R B F$ kernel with constant $(\boldsymbol{C}, \boldsymbol{G})=\left(2^{2.725}, 2^{2.725}\right)$ on the $2^{\text {nd }}$ feedback produces the better performance when compared to existing methods. In the Table 2. presents the average precision value on the baseline, on the results of testing techniques SVM RF kernel $R B F$, and on the results of the other method on each category of the Wang dataset. The average recall value is expressed in Table 3. While the comparison of the average F-measure value of each category obtained from the Wang dataset based on the baseline, the SVM RF with the RBF kernel with a constant $(\boldsymbol{C}, \boldsymbol{G})=\left(2^{2.725}, 2^{2.725}\right)$, and the state-of-the-art method is as stated in Fig. 11.

Based on the average precision value obtained that the SVM RF with the RBF kernel with $(\boldsymbol{C}, \boldsymbol{G})=\left(2^{2.725}\right.$, $2^{2.725}$ ) is $67.91 \%$. This result is still smaller than the average precision value that achieved by the other method except in [12]. Otherwise, if we consider the average precision value by category, it is found that the proposed technique has the best average precision value in a given category. In the Africans category, the proposed technique has the better average precision value when compared with the methods proposed by other researchers except in [29] and [13](Triangular Level 2 DBN). For the Horses category, the average precision value of the proposed technique has the better average precision value when compared to [13](Triangular Level 2 DBN), [9], [10], and [12], but less favorable when compared with the other methods.

The average recall value of the proposed technique is $80.06 \%$. The average recall value obtained by the proposed technique has a much better the recall value when compared to the existing method, even better than the technique proposed by [29]. The remarkable thing from the proposed technique is that it can increase the average recall value up to 2.72 times better when compared to the base line. The proposed technique in the recall value is much better than the existing method. The average recall value of the proposed technique also obtains the best average recall value for each category.

Table 2. Comparison of the Average Precision Value Compared to Other Methods Conduct by Category (\%)

\begin{tabular}{|c|c|c|c|c|c|c|c|c|c|c|c|c|}
\hline Category & $\begin{array}{c}\text { Baseline } \\
\text { HSV }\end{array}$ & $\begin{array}{l}\text { SVM RF } \\
\text { kernel } \\
\text { RBF } \\
\text { (Propose } \\
\text { d) }\end{array}$ & $\begin{array}{l}\text { Pardede, } \\
\text { J., et } \\
\text { al.,2018 } \\
\text { [29] }\end{array}$ & $\begin{array}{l}\text { Irtaza, } \\
\text { A., et } \\
\text { al., } \\
2014 \\
\text { [9] }\end{array}$ & $\begin{array}{l}\text { Irtaza } \\
\text { et al., } \\
2014 \\
{[10]}\end{array}$ & $\begin{array}{c}\text { Ashraf, } \\
\text { R., et } \\
\text { al., } \\
2015 \\
\text { [11] }\end{array}$ & $\begin{array}{l}\text { Rashno, } \\
\text { A., et al., } \\
2015 \text { [12] }\end{array}$ & $\begin{array}{c}\text { Ali, } \\
2016, \\
\text { Triangu } \\
\text { lar } \\
\text { (Level } \\
2 \text { DBN) } \\
\text { [13] }\end{array}$ & $\begin{array}{l}\text { Ali, 2016, } \\
\text { Triangula } \\
\text { r (Level } 2 \\
\text { SVM)[13] }\end{array}$ & $\begin{array}{c}\text { Ali, } \\
2016, \\
\text { Rectan } \\
\text { gular } \\
(2 \times 2 \\
\text { SVM)[1 } \\
3]\end{array}$ & $\begin{array}{c}\text { Hossai } \\
\text { n, M.S., } \\
\text { and } \\
\text { Islam, } \\
\text { M.R, } \\
2017 \\
{[14]}\end{array}$ & $\begin{array}{c}\text { Hossai } \\
\text { n, } \\
\text { M.S., } \\
\text { and } \\
\text { Islam, } \\
\text { M.R, } \\
2017 \\
{[14]}\end{array}$ \\
\hline Africans & 72.82 & 70.15 & 87.07 & 69.00 & 65.00 & 65.00 & 44.80 & 78.34 & 69.08 & 68.05 & 65.00 & 70.00 \\
\hline Beaches & 49.71 & 52.77 & 73.98 & 60.00 & 60.00 & 70.00 & 47.20 & 82.44 & 72.20 & 71.21 & 80.00 & 85.00 \\
\hline Buildings & 56.52 & 64.89 & 74.19 & 56.00 & 62.00 & 75.00 & 53.40 & 91.62 & 84.85 & 83.27 & 60.00 & 60.00 \\
\hline Buses & 72.26 & 84.70 & 92.56 & 89.00 & 85.00 & 95.00 & 73.40 & 96.00 & 97.50 & 96.01 & 100.00 & 100.00 \\
\hline Dinosaurs & 96.60 & 94.23 & 99.44 & 96.00 & 93.00 & 100.00 & 99.80 & 96.48 & 100.00 & 100.00 & 100.00 & 100.00 \\
\hline Elephants & 44.97 & 62.73 & 72.37 & 64.00 & 65.00 & 80.00 & 56.80 & 84.59 & 89.99 & 89.14 & 90.00 & 95.00 \\
\hline Flowers & 65.34 & 50.45 & 98.16 & 97.00 & 94.00 & 95,00 & 87.50 & 90.90 & 94.01 & 92.05 & 100.00 & 100.00 \\
\hline Horses & 89.72 & 81.33 & 97.30 & 66.00 & 77.00 & 90.00 & 70.70 & 78.02 & 86.38 & 86.22 & 95.00 & 100.00 \\
\hline $\begin{array}{c}\text { Mountain } \\
\mathrm{s}\end{array}$ & 49.89 & 51.32 & 76.87 & 57.00 & 73.00 & 75.00 & 39.30 & 87.52 & 82.85 & 81.41 & 70.00 & 80.00 \\
\hline Food & 61.38 & 66.53 & 83.97 & 86.00 & 81.00 & 75.00 & 61.00 & 90.63 & 85.88 & 83.06 & 80.00 & 85.00 \\
\hline Average & 65.92 & 67.91 & 85.59 & 74.00 & 75.50 & 82.00 & 63.39 & 87.65 & 86.27 & 85.04 & 84.00 & 87.50 \\
\hline
\end{tabular}


Table 3. The Comparison of the Average Recall Value Compared to Other Methods by Category (\%)

\begin{tabular}{|c|c|c|c|c|c|c|c|c|c|c|c|c|}
\hline Category & $\begin{array}{c}\text { Baselin } \\
\text { e HSV }\end{array}$ & $\begin{array}{l}\text { SVM RF } \\
\text { kernel } \\
\text { RBF } \\
\text { (Propose } \\
\text { d) }\end{array}$ & $\begin{array}{c}\text { Parded } \\
\text { e, J., et } \\
\text { al.,201 } \\
8 \text { [29] }\end{array}$ & $\begin{array}{l}\text { Irtaza, } \\
\text { A., et } \\
\text { al., } \\
2014 \\
\text { [9] }\end{array}$ & $\begin{array}{c}\text { Irtaza et } \\
\text { al., } 2014 \\
{[10]}\end{array}$ & $\begin{array}{c}\text { Ashraf, } \\
\text { R., et al., } \\
2015 \\
{[11]}\end{array}$ & $\begin{array}{c}\text { Rashno, } \\
\text { A., et } \\
\text { al., } \\
2015 \\
{[12]}\end{array}$ & $\begin{array}{c}\text { Ali, } \\
\text { 2016, } \\
\text { Triang } \\
\text { ular } \\
\text { (Level } \\
2 \mathrm{DBN} \text { ) } \\
{[13]}\end{array}$ & $\begin{array}{c}\text { Ali, } \\
2016, \\
\text { Triangu } \\
\text { lar } \\
\text { (Level } \\
2 \\
\text { SVM)[1 } \\
3]\end{array}$ & $\begin{array}{l}\text { Ali, 2016, } \\
\text { Rectangu } \\
\operatorname{lar}(2 \times 2 \\
\text { SVM)[13] }\end{array}$ & $\begin{array}{c}\text { Hossai } \\
\text { n, M.S., } \\
\text { and } \\
\text { Islam, } \\
\text { M.R, } \\
2017 \\
{[14]}\end{array}$ & $\begin{array}{c}\text { Hossain } \\
\text {, M.S., } \\
\text { and } \\
\text { Islam, } \\
\text { M.R, } \\
2017 \\
{[14]}\end{array}$ \\
\hline Africans & 54.11 & 92.78 & 48.26 & 13.00 & 13.00 & 13.00 & 10.90 & 15.67 & 13.82 & 13.70 & 13.00 & 14.00 \\
\hline Beaches & 7.44 & 57.89 & 5.07 & 12.00 & 12.00 & 14.00 & 11.90 & 16.49 & 14.44 & 14.24 & 16.00 & 17.00 \\
\hline Buildings & 16.67 & 80.22 & 13.41 & 12.00 & 12.40 & 15.00 & 13.00 & 18.32 & 16.97 & 16.65 & 12.00 & 12.00 \\
\hline Buses & 27.00 & 93.00 & 26.71 & 17.00 & 17.00 & 19.00 & 11.80 & 19.20 & 19.50 & 19.20 & 20.00 & 20.00 \\
\hline Dinosaurs & 44.44 & 99.89 & 51.70 & 19.00 & 18.60 & 20.00 & 19.90 & 19.30 & 20.00 & 20.00 & 20.00 & 20.00 \\
\hline Elephants & 12.33 & 67.33 & 9.44 & 13.00 & 13.00 & 16.00 & 13.40 & 16.92 & 18.00 & 17.83 & 18.00 & 19.00 \\
\hline Flowers & 17.78 & 74.22 & 17.09 & 19.00 & 18.80 & 19.00 & 17.50 & 18.18 & 18.80 & 18.50 & 20.00 & 20.00 \\
\hline Horses & 79.89 & 99.11 & 73.75 & 15.00 & 15.40 & 18.00 & 12.10 & 15.60 & 17.28 & 17.24 & 19.00 & 20.00 \\
\hline Mountains & 9.89 & 57.11 & 6.35 & 10.00 & 14.60 & 15.00 & 16.60 & 17.50 & 16.57 & 16.28 & 14.00 & 16.00 \\
\hline Food & 25.33 & 79.00 & 25.23 & 16.00 & 16.20 & 15.00 & 12.30 & 18.13 & 17.18 & 16.72 & 16.00 & 17.00 \\
\hline Average & 29.49 & 80.06 & 27.70 & 14.60 & 15.10 & 16.40 & 13.94 & 17.53 & 17.26 & 17.04 & 16.80 & 17.50 \\
\hline
\end{tabular}

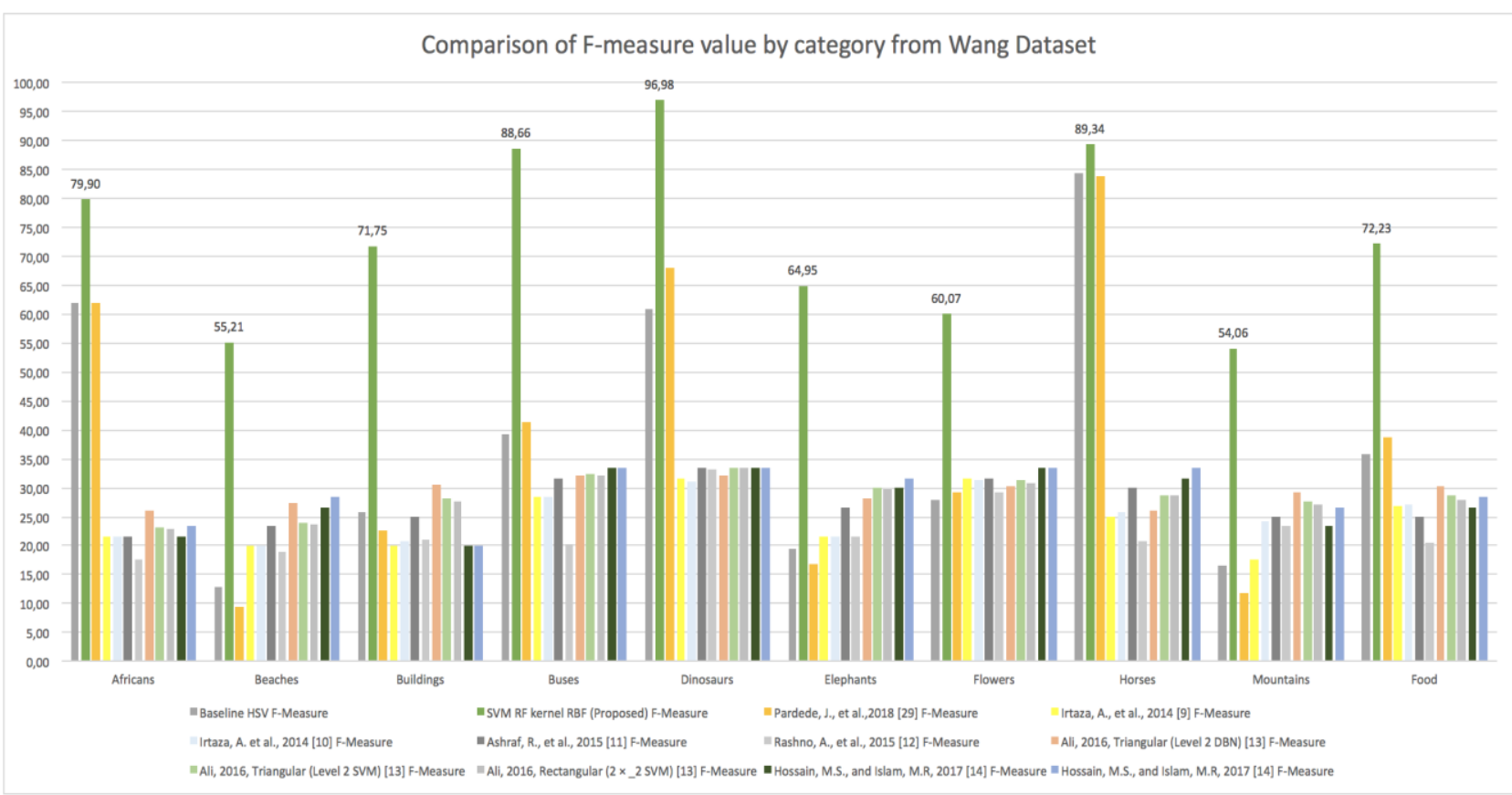

Fig. 11. Comparison of the average F-measure value (\%).

In the Dinosaurs and Horses category, the average recall value is greater than 99\%. It means that the SVM $\mathrm{RF}$ can retrieve mostly of existing imagery in its image database. While the existing method is generally only able to do retrieve images in the database only $20 \%$, except on [29]. This suggests that the proposed technique is capable of retrieving all imagery in the image database if each image has a uniform image feature vector distribution. The image feature vector distribution is as shown in Fig. 12. The distribution of image feature vector of the Dinosaurs category is represented by the color category 4, while the Horses category is represented by the color category 7. Based on the color distribution we can note that the Dinosaurs category and the Horses category are more clustered. The color distribution that more clustered 
can form a better support vector. It is very different for the category Beaches and Mountains. They are more spread so that not good for determining its support vector. Category Beaches are represented by the color category 1 . The distribution of the feature vector is the wider and only able to recall amount $57.89 \%$. While the distribution of the feature vector of the Mountains category is represented by the color category 8. It has the distribution of the feature vector that more spread. This is why the Mountains category has a very low recall value that only capable amount $57.11 \%$.

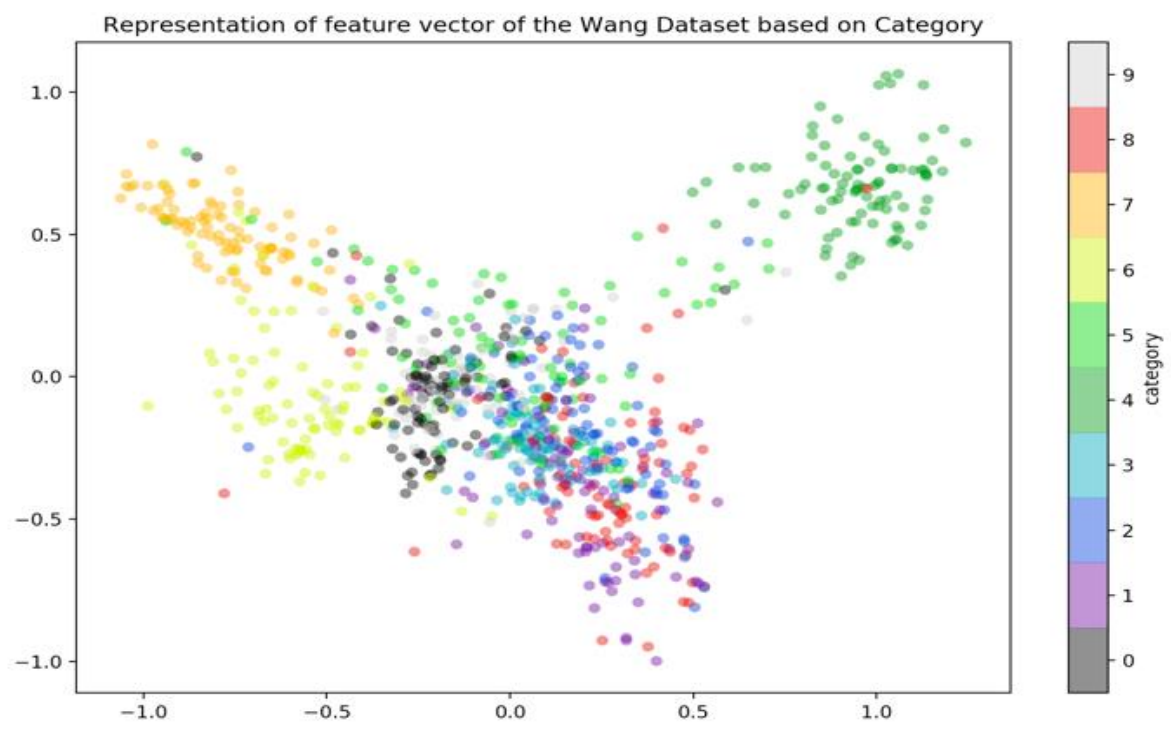

Fig. 12. Graphical representation of Wang dataset image distribution.

The magnitude of the increase in the recall value and the precision value has resulted increasing the average F-measure value of each category in the proposed technique. The proposed technique has the best performance in all categories when compared with all the methods that exist. The average F-measure value on each category is represented as in Fig. 11. In the proposed technique can improve the average F-measure value up to 1.80 times. It is better than the baseline of HSV Quantization, i.e. from $40.75 \%$ to $73.48 \%$. The proposed technique is much better than the method already available.

\section{Conclusion}

SVM can be implemented on RF techniques with target values labeled by the system as +1 for user feedback imagery, whereas unselected images will be labeled -1. In this research, we have conducted Wang dataset with various coefficient values i.e. both $(\boldsymbol{C}, \boldsymbol{G})$ for SVM $R G F$ kernel and various $\boldsymbol{C}$ values for the linear kernel. Based on the result of research, the best coefficient value for the $R B F$ kernel on the $2^{\text {nd }}$ feedback is $(\boldsymbol{C}$, $\boldsymbol{G})=\left(2^{2.275}, 2^{2.275}\right)$, while for the linear kernel on the $2^{\text {nd }}$ feedback is $\boldsymbol{C}=2^{5.725}$.

On Wang dataset SVM RF kernel $R B F$ with a constant value $(\boldsymbol{C}, \boldsymbol{G})=\left(2^{2.275}, 2^{2.275}\right)$ on the $2^{\text {nd }}$ feedback already provide the best precision, recall, F-measure, and accuracy value compared to the base line HSV Quantization with threshold 0.15 distance measurement Jeffrey Divergence. While the kernel linear only provide the better recall and F-measure value on the $2^{\text {nd }}$ feedback, while the precision value is smaller than the baseline. On the Wang dataset, the SVM RF with the linear kernel on the $2^{\text {nd }}$ feedback has the best precision value in the category of Buildings and Dinosaurs. While the value of recall and F-Measure produce better value in all categories. SVM RF with the linear kernel with $\boldsymbol{C}=2^{5.725}$ can increase the average recall value $121.48 \%$ and the average $F$-measure value is $52.73 \%$.

On the Wang dataset, the SVM RF with RBF kernel on the $2^{\text {nd }}$ feedback has the beast precision value in six categories, namely category Beaches, Buildings, Buses, Elephants, Mountains, and Foods. While the value of 
category is $99.89 \%$. The best F-Measure value is also obtained in the Dinosaurs category of $96.98 \%$. the SVM $\mathrm{RF}$ with $R B F$ kernel with $(\boldsymbol{C}, \boldsymbol{G})=\left(2^{2.275}, 2^{2.275}\right)$ could increase the average precision value on $3.02 \%$, the average recall value increased by $171.48 \%$, the average $F$-Measure value increased by $80.34 \%$, while the average accuracy value increased by $1.99 \%$. On Wang dataset, the SVM RF has the best performance with $R B F$ kernel with constants $(\boldsymbol{C}, \boldsymbol{G})=\left(2^{2.725}, 2^{2.725}\right)$. The RF SVM with $R B F$ kernel gives the best average recall value and average F-Measure value compared to the existing method. The number of given feedback images has no effect on the formation of the SVM RF model but is strongly influenced by the representation of user-provided feedback images. The feedback image that can form a good support vector will form the best RF SVM model. On the Wang dataset does not find an image that is a support vector, so there is no image that can be used to represent all images in one particular category.

\section{Acknowledgment}

This research has been supported by funding from LPDP Indonesia with number 20161141011055.

\section{References}

[1] Nalini, B. L., \& Malleswari, P. (2016). Review on content based image retrieval : From its origin to the new age. International Journal of Reserach Studies in Science, Engineering and Technology, 3(2), 18-41.

[2] Alkhawlani, M., \& Elmogy, M. (2015). Content-based image retrieval using local features descriptors and bag-of-visual words. International Journal of Advanced Computer Science and Applications, 6(9), 212-219.

[3] Khokher, A., \& Talwar, R. (2011). Image retrieval : A state of the art approach for CBIR. International Journal of Engineering Science and Technology (IJEST), 3(8), 6698-6704.

[4] Pinjarkar, L., \& Sharma, M. (2013). Content based image retrieval for trademark registration : A survey. International Journal of Advanced Reserach in Computer and Communication Engineering, 2(11), 4424-4430.

[5] Kumar, K. (2016). Content based image retrieval using gray scale weighted average method. (IJACSA) International Journal of Advanced Computer Science and Applications, 7(1), 1-6.

[6] Koskela, M., Laaksonen, J., \& Oja, E. (2001). Comparison of techniques for conten-based image retrieval. Proceedings of the 12th Scandinavian Conference on Image Analysis (SCIA 2001), 579-586.

[7] Shukla, J., \& Vania, J. (2014). A survey on CBIR features extraction techniques. International Journal of Engineering and Computer Science, 3(12), 9555-9559.

[8] Rao, M. B. (2011). Content based image retrieval using dominant color, texture and shape. International Journal of Engineering Science, 3(4), 2887-2896.

[9] Irtaza, A., Jaffar, M. A., \& Mahmood, M. T. (2014). Semantic image retrieval in a grid computing environment using support vector machines. The Computer Journal, 57(2), 205-216.

[10] Irtaza, A., Jaffar, M. A., Aleisa, E., \& Choi, T. S. (2014). Embedding neural networks for semantic association in content based image retrieval. Multimedia Tools and Applications, 72(2), 1911-1931.

[11] Ashraf, R., Bashir, Irtaza, K. A., \& Mahmood, M. (2015). Content based image retrieval using embedded neural networks with bandletized regions. Entropy, 17(6), 3552-3580.

[12] Rashno, A., Sadri, S., \& SadeghianNejad, H. (2015). An efficient content-based image retrieval with ant colony optimization feature selection schema based on wavelet and color features. Proceedings of the International Symposium on Artificial Intelligence and Signal Processing (AISP) (pp. 59-64).

[13] Ali, N., Bajwa, K. B., Sablatnig, R., \& Mehmood, Z. (2016). Image retrieval by addition of spatial information based on histograms of triangular regions. Computers and Electrical Engineering, 54, 539-550. 
[14] Hossain, M., \& Islam, M. (2017). A new approach of content based image retrieval using color and texture features. British Journal of Applied Science \& Technology, 21(3), 1-16.

[15] Deb, S. (2010). Using relevance feedback in bridging semantic gaps in content-based image retrieval. Proceedings of the Second International Conference on Advances in Future Internet.

[16] Caudill, J. (2009). Bridging the semantic gap in content-based image retrieval. Journal of Multimedia, 4(5), 277-283.

[17] Zhao, R., \& Grosky, W. I. (2002). Bridging the Semantic Gap in Image Retrieval. Department of Computer Engineering and Computer Science, University of Louisville, Kentucky.

[18] Zhou, X. S., Huang, T. S., \& Ave, N. M. (2001). Relevance feedback in image retrieval, a comprehensive review. ACM Multimedia Systems Journal.

[19] Zhou, X. S., \& Huang, T. S. (2003). Relevance feedback in image retrieval: A comprehensive review. Multimedia Systems, 8(6), 536-544.

[20] Saju, A., Mary, I. T. B., Vasuki, A., \& Lakshmi, P. S. (2014). Reduction of semantic gap using relevance feedback technique in image retrieval system. Proceedings of the 5th International Conference on the Applications of Digital Information and Web Technologies, ICADIWT 2014 (pp. 148-153).

[21] Zhang, J., Yang, Y., Zhuo, L., \& Diao, M. (2012). Image retrieval method using visual query suggestion and relevance feedback. IEEE 2012.

[22] Pinjarkar, L., Sharma, M., \& Mehta, K. (2012). Comparison and analysis of content based image retrieval systems based on relevance feedback. Journal of Emerging Trends in Computing and Information Sciences, 3(6), 833-837.

[23] Smeulders, A. W. M., Worring, M., Santini S., Gupta, A., \& Jain, R. (2000). Content-based image retrieval at the end of the early years. IEEE Transactions on Pattern Analysis and Machine Intelligence, 22(12), 1349-1380.

[24] Zinzuvadia, K. M., Tanawala, B. A., \& NBrahmbhatt, K. (2007). A survey on feature based image retrieval using classification and relevance feedback techniques. International Journal of Innovative Research in Computer and Communication Engineering (An ISO Certified Organization), 3297(1), 508-513.

[25] Pardede, J., \& Sitohang, B. (2017). Reduce semantic gap in content-based image retrieval. Advanced Science Letters, 23(11), 10664-10671.

[26] Sivakamasundari, G., \& Seenivasagam, V. (2012). Different relevance feedback techniques in CBIR: A survey and comparative study. Proceedings of 2012 International Conference on Computing, Electronics and Electrical Technologies, ICCEET 2012 (pp. 1115-1121).

[27] Rui, Y., Huang, T. S., \& Mehrotra, S. (1997). Content-based image retrieval with relevance feedback in MARS. Proceedings of International Conference on Image Processing, 2, 815-818.

[28] Ishikawa, Y., Subramanya, R., \& Faloutsos, C. (1998). MindReader: Querying databases through multiple examples. International Conference on Very Large Databases (VLDB), 218-227.

[29] Pardede, J., Sitohang, B., Akbar, S., \& Khodra, M. L. (2018). Re-weighting relevance feedback in HSV quantization for CBIR. Proceeding of the 19th IEEE/ACIS International Conference on Software Engineering, Artificial Intelligence, Networking and Parallel/Distributed Computing.

[30] Rui, Y., Huang, T. S., Ortega, M., \& Mehrotra, S. (1998). Relevance feedback: A power tool for interactive content-based image retrieval. IEEE Transactions on Circuits and Systems for Video Technology, 8(5), 644-655.

[31] Tong, S., \& Chang, E. (2001). Support vector machine active learning for image retrieval. Proceedings of the 9th ACM International Conference on Multimedia - MULTIMEDIA.

[32] Hoi, S. C. H., Zhu, J., \& Lyu, M. R. (2008). Semi-supervised SVM batch mode active learning with applications to image retrieval. Proceedings of IEEE Conferecence on Computer Vision and Pattern 
Recognition ACM Transactions on Information Systems (pp. 1-10).

[33] Bhagyalaksluni, I. A. (2014). A survey on content based image retrieval using various operators. Proceeding of 2014 IEEE International Conference on Computer Communication and Systems (ICCCS '14) (pp. 18-23).

[34] Shriwas, V. R., \& Raut, M. K. (2015). Content based image retrieval : A past, present and new feature descriptor. Proceedings of 2015 International Conference on Circuit, Power and Computing Technologies (ICCPCT).

[35] Shivhare, S. (2015). Content based image retrieval by using interactive relevance feedback technique A survey. International Journal on Recent and Innovation Trends in Computing and Communication, 3(7), 4641-4645.

[36] Pardede, J., Sitohang, B., Akbar, S., \& Khodra, M. L. (2017). Comparison of similarity measures in HSV quantization for CBIR. Proceedings of International Conference on Data and Software Engineering (ICoDSE).

[37] Bajaj, E. N. (2015). An approach for similarity matching and comparison in content based image retrieval system. IJ Information Engineering and Electronic Business, 5(9), 48-54.

[38] Campbell, C., \& Ying, Y. (2011). Learning with support vector machines. Synthesis Lectures on Artificial Intelligence and Machine Learning.

[39] Ngo, G. T., T. Q. \& Nguyen, D. D. (2016). Image retrieval with relevance feedback using SVM active learning. International Journal of Electrical and Computer Engineering (IJECE), 6(6), 3238-3248.

[40] Alzu'bi, A., Amira, A., \& Ramzan, N. (2015). Semantic content-based image retrieval: A comprehensive study. Journal of Visual Communication and Image Representation, 32(7), 20-54.

[41] Niranjanan, S., \& Gopalan, S. P. R. (2012). Performance Efficiency of quantization using HSV colour space and intersection distance in CBIR. International Journal of Computer Applications, 42, 48-55.

[42] Abdulmunem, A. (2016). Saliency guided local and global descriptors for effective action recognition. Computational Visual Media, 2(1), 97-106.

[43] Kavitha, C., Rao, D., \& Govardhan, D. (2011). Image retrieval based on color and texture features of the image sub-blocks. International Journal of Computer Applications, 15(7), 33-37.

[44] Singha, M. \& Hemachandran, K. (2012). Content based image retrieval using color and texture. Signal \& Image Processing : An International Journal (SIPIJ), 3(1), 39-57.

[45] Sangoh, J. (2008). Histogram-based color image retrieval. Psych221/EE362 Project Report, Stanford University, 1-21.

[46] Riaz, M., Pankoo, K., \& Jongan, P. (2009). Extracting color using adaptive segmentation for image retrieval. Proceedings of the 2009 International Joint Conference on Computational Sciences and Optimization, CSO (pp. 925-929).

[47] Di, B. (2007). An efficient image retrieval approach base on color clustering. Proceedings of the 3rd International Conference on Intelligent Information Hiding and Multimedia Signal Processing, IIHMSP (pp. 214-217).

[48] Ibraheem, C. M., \& Reddy, D. G. U. (2015). Content based image retrieval using HSV Color, shape and GLCM texture. International Journal of Advanced Research in Computer and Communication Engineering (IJARCCE), 4(10), 378-383.

[49] Murty, M. N. \& Raghava, R. (2016). Support Vector Machines and Perceptrons Learning, Optimization, Classification, and Application to Social Networks. Springer.

[50] VanderPlas, J. (2017). Python Data Science Handbook. O’Reilly.

[51] Abe, S. (2005). Support Vector Machines for Pattern Classification. Springer.

[52] Vapnik, V. N. (1998). Statistical learning theory. Adaptive and Learning Systems for Signal Processing, 
Communications and Control, 1-740.

[53] Hsu, C. J. L., \& Chang, C. C. (2008). A Practical Guide to Support Vector Classification and Regression.

[54] Salton, G. (1981). The Smart Environment for Retrieval System Evaluation - Advantages and Problem Areas.

[55] Cox, I. J., Miller, M. L., Omohundro, S. M., \& Yianilos, P. N. (1996). Target testing and the text pichunter bayesian multimedia retrieval system. Advances in Digital Libraries (ADL'96), 66-75.

[56] Thomee, B., \& Lew, M. S. (2012). Interactive search in image retrieval: A survey. International Journal of Multimedia Information Retrieval, 1(2), 71-86.

[57] Patil, V. I., \& Kotyal, S. (2015). Survey on content based image retrieval using color and texture features. International Journal of Advances in Electronics and Computer Science, 2(10), 1424-1429.

[58] Patil, S. (2012). A comprehensive review of recent relevance feedback techniques in CBIR. International Journal of Engineering, 1(6), 1-6.

[59] Hoi, S. C. H. H., Jin, R., Zhu, J., \& Lyu, M. R. (2008). Semi-supervised SVM batch mode active learning for image retrieval. Proceedings of IEEE Conference on Computer Vision and Pattern Recognition (CVPR2008) (pp. 1-7).

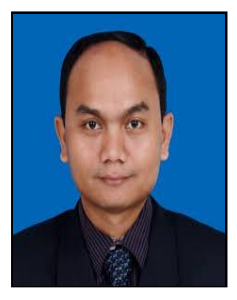

Jasman Pardede received the bachelor degree in science math from Universitas Andalas (Unand), Indonesia, in 2001, and the M.Eng degree in informatic engineering from Institut Teknologi Bandung (ITB), Bandung, Indonesia in 2005. Since 2005 until now, he is active as a lecturer at Institut Teknologi Nasional (Itenas) Bandung. He currently continuing his doctoral studies at Institut Teknologi Bandung (ITB), Bandung, Indonesia since 2016 in field image retrieval.

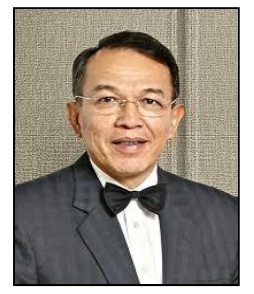

Benhard Sitohang is a professor in field of software engineering and knowledge. He received the bachelor degree from Institut Teknologi Bandung, Indonesia, in 1978. He received the master degree in software engineering from USTL-Montpellier, Montpellier, France in 1981, and he received doctoral degree from the same institute, in 1983. He currently works at Institut Teknologi Bandung (ITB) as a lecturer and researcher.

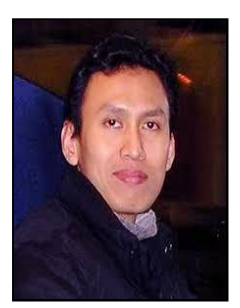

Saiful Akbar received the bachelor degree in Department of Informatics, School of Electrical and Informatics, Institut Teknologi Bandung (ITB), Bandung, Indonesia, in 1997, and the M.Eng degree in informatic engineering from Institut Teknologi Bandung (ITB), Bandung, Indonesia in 2002. He received the doctoral degree in engineering science from Johannes Kepler Universitat Linz - Austris, in 2007. Since 1998 until now, he is active as a lecturer at Institut Teknologi Bandung (ITB), Bandung, Indonesia. He currently works at Institut Teknologi Bandung (ITB) as a lecturer and assistant professor.

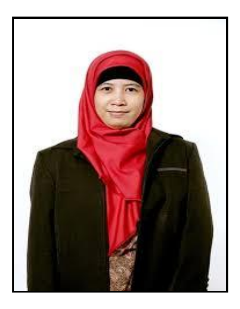

Masayu Leylia Khodra received the bachelor degree in Department of Informatics, School of Electrical and Informatics, Institut Teknologi Bandung (ITB), Bandung, Indonesia, in 1998, and the M.Eng degree in informatic engineering from Institut Teknologi Bandung (ITB), Bandung, Indonesia in 2006. She received the doctoral degree in the same institute, in 2012. Since 2008 until now, she is active as a lecturer at Institut Teknologi Bandung (ITB), Bandung, Indonesia. She currently works at Institut Teknologi Bandung (ITB) as a lecturer and assistant professor. 